this and foreign countries, show the comparative frequency of unusually located initial lesions and teaches us to be constantly watchful that no mistake be made.

of the following cases two were seen in the Dermatologic Clinic of Dr. Chas. N. Davis at the Pennsylvania Hospital, one in the Genitourinary Clinic of Dr. H. M. Christian at the Polyclinic Hospital, one in the Dermatologic Clinic of Dr. M. B. Hartzell at the University of Pennsylvania Hospital, and one in my own practice.

CASE 1.-Walter T., mulatto, age 26, clerk, born in Philadelphia. First seen in Surgical Dispensary of Pennsylvania Hospital on June 20, 1904. At that time he had a quarterdollar sized, elevated, sharply marginate, fungating, crusted, slightly indurated lesion on posterior surface of right index finger, over phalangeal-metacarpal articulation. This lesion was of six days' duration. No glandular or other specific symptoms were present. The treatment consisted of mild, local, antiseptic applications. The induration of this lesion was more marked on each successive visit. On July 17 the patient returned after an absence of ten days, with a typical roseolar eruption, general glandular enlargement, pharyngitis and pains in body. The patient had been referred to the Cutaneous Clinic, after a few dressings in the surgical dispensary, where the following history was discovered: On April 18 this man engaged in a fight, hitting his opponent in the teeth, causing an abrasion at the site of the present lesion. This abrasion subsequently healed, but on June 14, or eight weeks following this injury, the present lesion appeared. The secondaries appeared precociously in four and a half weeks.

CASE 2.-C. D., an Italian girl, aged 22, married, came to the Cutaneous Clinic at the Pennsylvania Hospital on May 1, 1905, showing the following: Just below left nipple, in areolar formed by glands of Montgomery, were two dime-sized, sharply marginate, raised, markedly indurated, crusted lesions, separated by about one inch of sound skin. On the right breast one other lesion of the same character and location was seen. The duration was fifteen days. The only subjective sign was pain all over the body. No objective sign of syphilis was present. The patient was seen three times during the next ten days with no new developments except an increase in the button-like induration of the lesions and in severity of muscular and osseous pain. After an interval of almost three weeks the patient returned on June 27 with . macular eruption of one week's duration, covering the entire body and face; numerous mucous patches of lips, tongue, sof $\lrcorner$ palate and tonsils were present, also general glandular enlargement and pains in entire body. Unfortunately no history was obtainable in this case. Her only child, a girl of 11 months, was apparently in perfect health, although still breast-fed.

CASE 3.-F. P., male, aged 30, came to my office May 29, 1905, complaining of a urethral discharge of ten days' duration. On examination an irreducible phimosis was found, also a profuse muco-purulent discharge. Of still greater interest, on palpating the penis, a hazelnut sized, firmly indurated, button-like mass was found one and a half inches behind the meatus, on the roof of the urethra and partially surrounding the same, this latter mass being of three weeks' duration. After cleansing the foreskin with potassium permanganate solution, the two glass test was tried, the first glass only showing cloudy urine with shreds. The only subjective sign was bilateral enlargement of inguinal glands. No primary incubation period could be determined, for the man was extremely promiscuous in his intercourse. On June 19 the patient again appeared in my office, after an absence of three weeks, with a profuse, ham colored, multiform eruption, chiefly small, flat, papular in type, general glandular enlargement, husky voice, pharyngitis, slight thinning of hair, and generalized pains in bones and joints. These secondary symptoms appeared ten days before this present visit. No microscopic examination having been made for the diplococcus of Neisser, a superadded gonorrhea can not be positively stated.

CASE 4.-S. M., male, aged 22, came to Genitourinary Clinic at the Polyclinic Hospital on Aug. 15, 1905, with a dime sized, sharply marginate, crusted, slightly raised indurated lesion, slightly to left of median line of lower lip. The duration was ten days. The incubation period was indefinite. There were no other signs of beginning syphilis. On September 12 the patient returned, after an absence of five weeks, with a marked pharyngitis, the post-cervical, submaxillary and inguinal glands were enlarged, and a faint macular eruption of one day's duration was on chest, abdomen and back. The generalized eruption in this case followed a secondary incubation period of just six weeks.

CASE 5.-Charles T., aged 42, blacksmith. This patient first came to the Cutaneous Clinic of the University of Pennsylvania Hospital on Feb. 23, 1906, complaining of a large swelling of the lower lip. On the vermilion of the lower lip just to the left of the median line, a quarter-dollar sized densely indurated, slightly crusted, raised, button-like lesion was found. The post-cervical and submaxillary glands were enlarged and an extragenital chancre was diagnosed. The man stated he had no eruption on the body. As in all cases, not trusting to the patient's own observation, we made the man disrobe. One glance at the patient's body was sufficient to see a generalized macular eruption over arms, legs, trunk, and a few lesions of the same character on the forehead. The throat showed a pharyngitis, with small mucous patches of postpharyngeal arches. The exact primary or secondary incubation period, or the duration of the initial lesion and secondary eruption could not be determined. The patient stated that the lesion had appeared on the lip three weeks ago; probably twice that length of time would be nearer the truth. The macular eruption could have had a duration of but a few days. This man, being separated from his wife, was in more danger of being exposed to contagion.

Only those cases have been reported in this series in which the diagnosis was proved by the appearance of the secondary eruption and concomitant signs of syphilis.

During this same period three other cases were seen, in each of which the provisional diagnosis of extragenital chancre was made, two of these cases having indurated, single, lesions on the fingers and in one case on the upper lip. Unfortunately, as each of these patients made but the one visit, our provisional diagnosis could not be conclusively proved.

332 South Seventeenth Street.

\section{A CASE OF PRIMARY SYPHILITIC INFECTION}

$$
\text { IN THE NOSE.* }
$$

JAMES T. CAMPBELL, M.D.

Professor of Otology, Rhinology and Laryngology in the Postgraduate Medical School. CHICAGO.

Chancre of the nose is one of the rarest of lesions. Krefting ${ }^{\mathbf{1}}$ gives statistics of 2,916 cases of chancres, of which 539 were instances of extragenital infection, and Salsotto $^{2}$ records 201 cases of extragenital chancre with no case of primary nasal infection. Bulkley ${ }^{3}$ gives a table of 9,058 cases of extragenital chancre in which primary infection of the nose occurred ninety-five times.

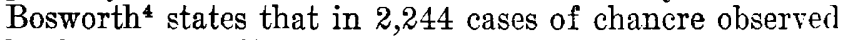
by Bossereau, Clerq, Lefort, Fornier and Ricord, the primary lesion was found twice in the nose. Le Bart ${ }^{5}$ reports thirty-seven cases of primary nasal chancre, of which twenty-one were external and sixteen within the nostril.

The earliest recorded ease I can find is that of Mc-

* Read before the Chicago Laryngological and Otological Society.

1. Archiv. f. Derm. u. Syph., 1894, vol. xxvi, p. 167.

2. Slflomi Extragenatali et Epidermie di Sifillde, Brochure, Turin, 1892.

3. Syphilis in the Innocent.

4. A Treatment on the Nose and Throat, 1889 .

5. These dé Paris, 1894. 
Carthy. ${ }^{6}$ Spencer Watson ${ }^{7}$ reported a case of a nurse in attendance on a woman who gave birth to a syphilitic child. The sore could not be distinctly seen, on account of the swelling within the nostril. Severe pain, fever and mental depression was followed by the ordinary symptoms of secondary syphilis. The vehicle of infection in all probability, was the patient's own finger.

Intranasal inoculations occur most frequently in the lower and anterior part of the nasal septum, next in frequency on the ala, and then, as in the case I am about to report, on the inferior turbinated body. The location modifies the chancre's appearance. On the septum it displays a flat, reddish or greenish, fungiform mass with indurated circumference. The surrounding mucous membrane is, to a greater or less degree, swollen, and there flows from the ofttimes stenosed nostril, a bloody, fetid discharge. When the ala is involved the infiltration and induration often causes it to be of a cartilaginous consistency. When the inferior turbinated is primarily inoculated, the appearance resembles, mostly, a severe localized influenza, or a fibrinous or diphtheritic rhinitis. Usually the submaxillary, sublingual and preauricular glands early show marked indolent swelling.

Characteristic of this infection is an aggravated general febrile disturbance, malaise and depression of spirits. The chancre may be mistaken for an abscess of, or injury to, the septum; for a furuncle, to which for a time, it is not dissimilar; for vaccine inoculation; for tubercular ulceration; for malignant disease.

The existence of an ulcer in the nose with a peculiar hard base and granular surface, bleeding easily on touch and of limited extent, would suggest tuberculosis, malignant disease or syphilis. Tubercular ulceration occurs only as secondary to a pulmonary deposit and examination of the discharge would show characteristic bacilli. In malignant disease glandular enlargement is a late development, and the progress of the disease is liable to be accompanied by profuse epistaxes. In syphilis the chancre is followed shortly by characteristic secondary symptoms.

\section{CASE REPORT.}

History.-On Oct. 19, 1905, a surgeon in perfect health circumcised a patient, on whose prepuce was a large indurated chancre.

On Deeember 16, nearly two months later, he first noticed stuffiness of the right nostril, and headache from brow across vertex to occiput. This was accompanied by malaise, anorexia, chilly sensations and constipation, a condition from which he never before had suffered. For a period of two weeks his temperature ranged from 100 to $100.2 \mathrm{~F}$. He had worked very hard for a few weeks prior to December 16, and he attributed his condition to overwork and a grip-like attack. During Christmas week he visited his old home, but the rest did not improve his condition.

Examination.-I first saw him on Dec. 28, 1905. At that time I made the notation, "Superficial necrosis of the mucous membrane covering the anterior end of the right inferior turbinate body; the fibrinous membrane, when raised, revealed an ulcerating bleeđing surface." Neither suprarenalin 1 to 1,000 nor 10 per cent. cocain solution caused any appreciable blanching or shrinking of the turbinated body. Lacrimation was present on the right side and one gland below the angle of the right jaw was enlarged and tender.

The condition resembled fibrinous rhinitis, but it was limited to the inferior turbinated body and there was no ichorous diseharge from the nostril. The swelling practically occluded the nostril, which is narrow on account of septal deviation, the result of traumatism in childhood.

The treatment employed was a cleansing alkaline spray followed by sprays of peroxid of hydrogen and lime water.

6. These de Paris, 1844.

7. If edical Times and Gazette, 1881, vol. 1, p. 428 .
Course of Disease.-On Jan. 8, 1906, eighty days after the probable inoculation, a macular rash appeared on the abdomen. Four days later, when I was shown the rash, I made a positive diagnosis of syphilis and was confirmed in this by Dr. Joseph Zeisler and Dr. T. Melville Hardie. The "leanham" macular and papular rash, more marked on his chest and abdomen, covered practically the whole body with the exception of the exposed portions of head and hands. There were papules at the base of the uvula, but no soreness or congestion of the fauces and no glandular enlargement or soreness excepting the before-mentioned submaxillary gland. Disappearance of the fibrinous membrane and healing of the ulceration took place under the simple sprays, before constitutional symptoms appeared. Coincident with the appearance of the rash, the headache ceased and the general feeling of wellbeing began.

On diagnosis of syphilis being made, inunctions of mercurial ointment, one dram, at bedtime were begun and after six rubbings all evidence of the disease had disappeared.

\section{A CASE OF MULTIPLE CHANCRES.}

\section{S. R. MALLORY KENNEDY, M.D., A.A.S. Public Health and Marine-Hospital Service. PENSACOLA, FLA.}

Cases of multiple chancres are rare. Chiefly because of their infrequency they are often unrecognized, and so the report of a single case, though adding nothing to the present-day knowledge of the subject, may call attention to the existence of a condition which might otherwise be overlooked. It is for this reason that I report the first case that, to my knowledge, has been treated in this section of the country.

Patient.-J. I. C., aged 22, presented a good family history.

History.-He had never been sick in his life except for an acne which bothered him but little, and for which he had done nothing. He first came under my care in August, 1904, when I treated him for a chronic form of gonorrhea. This, though stubborn, yielded after a time and the patient, after many tests, was discharged as cured. I did not see him again until August, 1905.

Examination.-On making an examination $I$ found a purulent discharge about the urinary meatus, and a single, enlarged, painless, indurated, freely movable gland in the left inguinal region. The discharge, however, did not come from the meatus, but from beneath the prepuce, which was slightly swollen, tending to phimosis. On inserting a small, blunt probe several rough surfaces could be detected, and on questioning the patient I was somewhat surprised to learn that he had not had sexual intercourse for more than a month before noticing the discharge.

Treatment.- Realizing that with the existing phimosis local treatment would amount to little or nothing, I advised an in. cision through the dorsum of the prepuce to expose the glans and to aid in making topical applications. He consented, and on the following day, with Iocal anesthesia-ethyl chlorid and a 2 per cent. solution of cocain-the incision was made, disclosing nine ulcers. Each lesion presented a typical picture. Their edges were sloping (cup shaped), they had a firm, eartilaginous, or woody feel. Their bases were covered with a thin, scanty, sero-purulent discharge which had no offensive odor. Each lesion was fully developed, separate and distinct. There was no tendency whatever to coalesce.

I was convinced that the picture before me was one of multiple chancres, and, realizing that all nine could be gotten rid of by a circumcision (all being located on the glandular surface of the prepuce), the primary incision was continued under cocain and a circumcision was done. The operation was followed by some little swelling and pain, but no more than would ordinarily occur after the performance of this operation on a perfectly healthy penis, the wound healing by first intention.

Bacteriologic Examination.-Smears from the nine lesions were made and examined for the bacillus of chancroid (bacillus of Ducrey) with negative results. Further tests were made 